\title{
The effect of prolonged aerobic exercise on serum adipokine levels during an ultra-marathon endurance race
}

\author{
Nikolaos D. Roupas, ${ }^{1}$ Irene Mamali, ${ }^{2}$ Spyros Maragkos, ${ }^{3}$ Lydia Leonidou, ${ }^{4}$ \\ Anastasia K. Armeni, ${ }^{1}$ George K. Markantes, ${ }^{1}$ Athanasios Tsekouras, ${ }^{2}$ \\ George C. Sakellaropoulos, ${ }^{5}$ Kostas B. Markou, ${ }^{2}$ Neoklis A. Georgopoulos ${ }^{1}$
}

${ }^{1}$ Division of Reproductive Endocrinology, Department of Obstetrics and Gynecology, ${ }^{2}$ Division of Endocrinology, Department of Internal Medicine, ${ }^{3}$ Department of Orthopedics, ${ }^{4}$ Division of Infectious Diseases, Department of Internal Medicine, ${ }^{5}$ Department of Medical Physics, University of Patras Medical School, Patras, Greece

\begin{abstract}
OBJECTIVE: To evaluate the effect of prolonged intensive aerobic exercise and acute energy deficit (180 $\mathrm{km}$ ultra-marathon race) on serum leptin, adiponectin, resistin and visfatin levels and their association and interaction with serum cortisol and insulin levels in highly trained ultra-endurance runners. DESIGN: The study included 17 highly trained ultra-endurance male athletes (mean age 51.29 \pm 6.84 years and body mass index (BMI) $23.51 \pm 1.90$ ) participating in the $5^{\text {th }}$ Olympian Race held in Greece on May 2010. Anthropometric values were assessed; Serum cortisol, insulin, leptin, adiponectin, resistin and visfatin levels were measured at baseline, post-exercise and 20 hours after the end of the race. RESULTS: All hormonal values of the post-exercise and recovery status were corrected for plasma volume changes. The estimated energy deficit during the ultra-endurance event was about $5000 \mathrm{Kcal}$. At the end of the race serum resistin levels were elevated $(p<0.001)$ and serum leptin levels were reduced $(p<0.001)$ and failed to reach pre-exercise levels, although showing a tendency towards restoration. No significant changes were noted in serum adiponectin and visfatin levels. CONCLUSIONS: Ultra-endurance aerobic exercise and acute negative energy balance lead to an up-regulation of serum resistin levels and a down-regulation of serum leptin levels.
\end{abstract}

Key words: Adiponectin, Adipose tissue, Athlete, Leptin, Resistin

Address for correspondence:

Neoklis A. Georgopoulos, Department of Obstetrics and Gynecology, Division of Reproductive Endocrinology,

University of Patras Medical School, University Hospital, 26500 Patras, Greece, Tel.: +302610999835, Fax:

+302610993854, e-mail: neoklisg@hol.gr

Received 10-09-2012, Accepted 16-04-2013

\section{INTRODUCTION}

Physical exercise represents a powerful stimulus to homeostasis, disturbs the energy balance, triggers metabolic changes and activates regulatory responses, establishing a new dynamic equilibrium. The maintenance of homeostasis involves adaptive changes in 
central and peripheral regulatory mechanisms, a new steady state of glucoregulatory mechanisms, activation of the hypothalamic-pituitary-adrenal (HPA) axis and changes of the secretory role of adipose tissue. The hormonal and metabolic response depends on the intensity, the duration, the type of exercise and the individual training status. ${ }^{1}$

Attention is being increasingly focused on the role of adipose tissue as an active endocrine organ releasing a number of adipocyte-specific factors known as adipokines. Adipokines are involved in glucose homeostasis, energy balance, insulin action, reproductive function and inflammation process. ${ }^{2}$

Specifically, leptin is involved in the regulation of food intake, energy expenditure and insulin resistance (IR). ${ }^{2}$ Adiponectin is secreted exclusively from adipose tissue and is detected at high levels in serum, while also being involved in metabolism, IR, cardiovascular protection and inflammatory process. ${ }^{2}$ Visfatin is expressed in a variety of cell types and tissues, including adipose tissue, and is regarded as a novel insulin-mimetic, fat-secreted regulatory factor involved in pro-inflammatory and immunomodulating processes. ${ }^{3}$ Finally, resistin has been described as a product of the adipose tissue, participating in the pathogenesis of IR, adipogenesis and inflammation. ${ }^{2}$

The influence of prolonged intensive aerobic exercise has been studied in regard to serum leptin response and regulation, ${ }^{4}$ while no data exist regarding the homeostasis of adiponectin, visfatin and resistin during strenuous exercise of long duration.

The aim of the present study was to evaluate the effect of prolonged intensive aerobic exercise and acute energy deficit (ultra-marathon endurance race of 180 $\mathrm{km}$ distance) on serum leptin, adiponectin, resistin and visfatin levels. Moreover, the study attempted to assess the association of the specific adipokines with serum cortisol and insulin levels in highly trained marathon runners, considering the reported data on their relationship with stress response and insulin resistance. ${ }^{2,3}$ Finally, the results of this study aimed at providing further evidence on the role of the specific adipokines in energy homeostasis and the response to exercise stress.

\section{MATERIALS AND METHODS}

The present study included 17 highly trained ultramarathon endurance male athletes participating in the $5^{\text {th }}$ Olympian Race held in Greece on 28 and 29 of May, 2010. The Olympian Race is an ultra-marathon endurance race (180 $\mathrm{km}$ of continuous running) held every two years in Greece. The goal of the participants is to complete the race within the 28-hour time limit. The race starts on Friday at $1430 \mathrm{hrs}$ in the Ancient Stadium of Nemea and successful competitors reach the Ancient Stadium of Olympia on Saturday no later than at $1830 \mathrm{hrs}$.

The trail races have special rules and specifications and comprise the largest possible part of the route on roads and paths over mountainous terrain and the fewest possible paths on tar roads. The athletes have free access to food and drink (including carbohydrate-rich foods and drinks) at 20 checkpoints throughout the race.

Sixty (60) ultra-endurance athletes participated in the $5^{\text {th }}$ Olympian Road. All of them were invited to be enrolled in the study, but only 25 agreed to participate. Among the 60 athletes who started the race, only 40 managed to reach the finishing point within the acceptable time period of $28 \mathrm{hrs}$ and 17 of these were participants of the present study.

All athletes participated voluntarily in every part of the study, providing samples or data for each of the variables measured and informed consent was obtained. The anthropometric features and the training history of the participants are shown in Table 1.

The athletes were evaluated three times: on the morning of the race (baseline values, at $0800 \mathrm{hrs}$ before breakfast - fasting samples), at the finishing line (post-exercise values, around $1500 \mathrm{hrs,} \mathrm{within} \mathrm{a}$ few minutes after the end of the running race - nonfasting samples) and on the next morning (recovery values, at 08:00hrs before breakfast, within 17-22 hrs after the end of the race - fasting samples).

The study protocol included noninvasive clinical and laboratory investigations as well as the completion of a questionnaire. The clinical evaluation included height and weight measurements. Height was assessed with a Martin-type anthropometer and was recorded as the mean of two consecutive measurements. Body 
Table 1. Anthropometric characteristics at baseline and post-exercise and training history of highly trained ultra-endurance athletes

\begin{tabular}{lcc}
\hline Parameters & $\begin{array}{c}\text { Baseline } \\
\text { values } \\
(\mathbf{n = 1 7})\end{array}$ & $\begin{array}{c}\text { Post-exercise } \\
\text { values } \\
(\mathbf{n}=\mathbf{6})\end{array}$ \\
\hline Age (years) & $51.3 \pm 6.8$ & \\
Body weight $(\mathrm{Kg})$ & $71.7 \pm 6.2$ & $68.8 \pm 7.2^{\mathrm{a}}$ \\
BMI $\left(\mathrm{Kg} / \mathrm{m}^{2}\right)$ & $23.5 \pm 1.9$ & $22.4 \pm 0.8^{\mathrm{a}}$ \\
Body fat $(\%)$ & $15.4 \pm 3.7$ & $13.5 \pm 3.1$ \\
Body fat mass $(\mathrm{Kg})$ & $11.1 \pm 3.2$ & $9.3 \pm 2.4^{\mathrm{a}}$ \\
Duration of training (years) & $18.5 \pm 12.3$ & \\
Intensity of training (hours/week) & $10.2 \pm 4.5$ & \\
Competitions per year & $6.6 \pm 6$ & \\
\hline
\end{tabular}

Values are expressed as mean \pm SD

a: $p<0.05$ vs. baseline values.

composition and body weight were evaluated using a portable apparatus (Tanita, BC-418MA, TANITA UK LTD), calculating body fat and total body water, via Bioelectrical Impedance Analysis and estimation of skin fold of seven different body shapes.

All athletes completed a questionnaire involving personal data and training history (age at onset and total duration of training, usual weekly intensity of training, number of participations in endurance races per year).

Blood samples were obtained from forearm veins. After centrifugation ( $3000 \mathrm{rpm}$ for 15 minutes), serum samples were stored at $-20{ }^{\circ} \mathrm{C}$ until the assay.

All hormonal values of the post-exercise and recovery status were corrected for plasma volume changes, following the equation suggested by Beaumont. ${ }^{5}$ This equation estimates plasma volume changes based on hematocrit measurements.

\section{Assays}

Serum resistin levels were measured using a commercial enzyme immunoassay kit (Cat. No DRSN00, R\&D Systems, Inc. Minneapolis, USA).

Serum leptin levels were measured using a commercial enzyme immunoassay kit (Cat. No DLP00, R\&D Systems, Inc. Minneapolis, USA).

Serum adiponectin levels were measured using a commercial enzyme immunoassay kit (Cat. No DRP300, R\&D Systems, Inc. Minneapolis, USA).

Serum visfatin levels were measured using a commercial enzyme immunoassay kit (Cat. No EK-003-80, Phoenix Pharmaceuticals, Inc. Burlingame, USA).

Serum insulin and cortisol levels were measured by electrochemiluminescence quantitation (Elecsys 2010, Roche Diagnostics, Tokyo, Japan).

The detailed technical characteristics of the chosen assays for each one of the studied hormone are presented in Table 2.

\section{Statistics}

All statistical procedures were performed using SPSS 18.0 for Windows (IBM SPSS Statistics, IBM software). Test for normality was done using the Kolmogorov-Smirnov test. Anthropometric features were expressed as mean $\pm \mathrm{SD}$ and hormonal values as median and interquartile range (25th and 75th).

Bivariate correlations among hormonal features and between hormonal and anthropometric features were assessed with Pearson product-moment correlation and Spearman's rank order correlation (for normally and non-normally distributed variables, respectively). Correlations with a critical value of

Table 2. Technical characteristics of the chosen assays for each one of the studied hormones

\begin{tabular}{lcccc}
\hline Hormone & Method & Detection limit & Intra-assay precision $($ CV \%) & Inter-assay precision $($ CV \%) \\
\hline Resistin & ELISA & $0.026 \mathrm{ng} / \mathrm{ml}$ & $<3.8$ & $<7.8$ \\
Leptin & ELISA & $7.8 \mathrm{pg} / \mathrm{ml}$ & $<3$ & $<3.5$ \\
Adiponectin & ELISA & $0.246 \mathrm{ng} / \mathrm{ml}$ & $<2.5$ & $<5.8$ \\
Visfatin & ELISA & $2.25 \mathrm{ng} / \mathrm{ml}$ & $<10$ & $<15$ \\
Insulin & ECLIA quantitation & $0.2 \mu \mathrm{U} / \mathrm{ml}$ & $<1.5$ & $<4.1$ \\
Cortisol & ECLIA quantitation & $0.018 \mu \mathrm{g} / \mathrm{dl}$ & $<1.5$ & $<2.1$ \\
\hline
\end{tabular}

ELISA: Enzyme-linked immunosorbent assay, ECLIA: Electrochemiluminescence immunoassay. 
$\mathrm{p}<0.05$ were considered significant. Stepwise linear regression analysis was used to ascertain the independent predictive value and impact of each parameter proved to significantly correlate.

Comparison between baseline, post-exercise and recovery hormonal values was performed with repeated-measures one-way analysis of variance (ANOVA) for normally distributed variables and with the Friedman test for non-normally distributed variables. Bonferroni adjustment for multiple comparisons was applied. Comparison between baseline and post-exercise features regarding body composition was performed using the paired-samples t-test. Twotailed statistical significance was set at 5\%.

\section{RESULTS}

\section{Changes in anthropometric values}

The anthropometric features were assessed at baseline for all 17 participants and at the end of the race for 6 of them (Table 1). The comparison showed a significant reduction in BMI, body weight and body fat mass ( $\mathrm{p}<0.001, \mathrm{p}<0.001$ and $\mathrm{p}<0.05$, respectively) and a marginal, but not significant, reduction in body fat percentage $(p=0.082)$.

\section{Changes in hormonal values (comparison among baseline, post-exercise and recovery values)}

At the end of the $180 \mathrm{~km}$ race resistin levels were significantly higher compared to baseline values (Table $3, p<0.001)$. At the recovery phase resistin levels were reduced compared to post-exercise levels (Table 3 , $\mathrm{p}<0.05$ ), but remained significantly higher compared to baseline values (Table 3, $\mathrm{p}<0.05$ ).

Furthermore, at the end of the $180 \mathrm{~km}$ race leptin levels were significantly lower compared to baseline values (Table $3, \mathrm{p}<0.001$ ). If leptin values are expressed as the ratio per kilogram $(\mathrm{kg})$ of body fat (leptin/kg of body fat), a significant reduction at the end of the race is documented as well $(p<0.001)$. At the recovery phase serum leptin levels were higher compared to post-exercise levels (Table $3, \mathrm{p}<0.001$ ), but remained significantly lower compared to baseline values (Table $3, \mathrm{p}<0.001$ ).

No differences were documented between baseline
Table 3. Hormonal features of participants at baseline, post-exercise and at recovery*

\begin{tabular}{lccc}
\hline & $\begin{array}{c}\text { Baseline } \\
(\mathbf{n}=\mathbf{1 7})\end{array}$ & $\begin{array}{c}\text { Post-exercise } \\
(\mathbf{n}=\mathbf{1 7})\end{array}$ & $\begin{array}{c}\text { Recovery } \\
(\mathbf{n}=\mathbf{1 7})\end{array}$ \\
\hline $\begin{array}{l}\text { Resistin } \\
(\mathrm{ng} / \mathrm{ml})\end{array}$ & 7 & $77.9^{\mathrm{a}}$ & $16.1^{\mathrm{a}, \mathrm{b}}$ \\
Leptin & 5.68 .6 & 10.792 .1 & 19.075 .3 \\
$(\mathrm{ng} / \mathrm{ml})$ & 1.6 & $0.2^{\mathrm{a}}$ & $0.7^{\mathrm{a}, \mathrm{b}}$ \\
Adiponectin & 12 & 0.10 .3 & 0.41 .3 \\
$(\mu \mathrm{g} / \mathrm{ml})$ & 8.1 & 8.2 & $7.8^{\mathrm{b}}$ \\
Visfatin & 4.612 .4 & 5.812 .5 & 5.110 .3 \\
$(\mathrm{ng} / \mathrm{ml})$ & 30.9 & 32.8 & 35.9 \\
Cortisol & 26.544 & 2638.7 & 30.342 .5 \\
$(\mu \mathrm{g} / \mathrm{dl})$ & 17.6 & $39.77^{\mathrm{a}}$ & $19.20^{\mathrm{b}}$ \\
Insulin & 15.220 .4 & 26.348 .9 & 17.925 .6 \\
$(\mu \mathrm{IU} / \mathrm{ml})$ & 5.1 & $2.3^{\mathrm{a}}$ & $7.5^{\mathrm{b}}$ \\
\hline $\mathrm{H}$ & 4.117 .1 & 1.54 .2 & 3.232 .4 \\
\hline
\end{tabular}

*Hormonal values are expressed as median and interquartile range $\left(25^{\text {th }}-75^{\text {th }}\right)$.

a: $p<0.05$ vs. baseline value, $b: p<0.05$ vs. post-exercise value Bonferroni adjustment for multiple comparisons was applied.

and post-exercise serum adiponectin and visfatin levels (Table 3). At the recovery phase, although adiponectin levels were lower compared to the postexercise values (Table $3, \mathrm{p}<0.05$ ), no difference was documented in comparison to the baseline values.

In addition, post-exercise cortisol levels were significantly higher compared to baseline values (Table 3 , $\mathrm{p}<0.001)$. At the recovery phase serum cortisol levels were lower compared to post-exercise levels (Table 3, $\mathrm{p}<0.001$ ) and returned to baseline levels (Table 3).

Finally, post-exercise insulin levels were significantly reduced compared to baseline values (Table $3, p<0.05)$. At the recovery phase, insulin levels were higher compared to post-exercise levels (Table 3 , $\mathrm{p}<0.05$ ) and returned to baseline levels (Table 3 ).

\section{Correlations}

Correlations between anthropometric features and hormonal values at baseline

Baseline leptin levels were positively correlated with BMI $(r=0.519, p=0.029)$ and body fat mass $(\mathrm{r}=0.534, \mathrm{p}=0.027)$, while a marginal but not significant positive correlation was documented between serum leptin levels and body fat percentage $(r=0.447$, $\mathrm{p}=0.072$ ). 
Moreover, baseline insulin levels were positively correlated with BMI $(\mathrm{r}=0.518, \mathrm{p}=0.033)$, while a marginal but not significant positive correlation was documented between insulin levels and age $(r=0.441$, $\mathrm{p}=0.077$ ).

Finally, baseline cortisol levels were inversely correlated with BMI $(\mathrm{r}=-0.530, \mathrm{p}=0.029)$.

\section{Correlations between hormonal values}

At baseline, serum leptin levels were strongly positively correlated with serum insulin levels $(\mathrm{r}=0.518$. $\mathrm{p}=0.033$ ), with no change of their correlation after control of the effect of body fat mass $(r=0.534$, $\mathrm{p}=0.027)$. In addition, the change in serum leptin levels $(\Delta$ leptin $=$ leptin post-exercise-baseline leptin $)$ and the change in serum insulin levels $(\Delta$ insulin $=$ insulin post-exercise-baseline insulin) were strongly positively correlated $(r=0.561, p=0.019)$. On the other hand, no significant correlations were documented among measured adipokine, insulin and cortisol levels.

\section{Stepwise regression analysis}

Stepwise regression analysis was performed in order to ascertain the independent predictive value and the impact of serum insulin levels and body fat mass on serum leptin levels. Stepwise regression analysis showed that serum insulin levels could predict $35.3 \%$ of serum leptin levels $(\mathrm{R}=0.594, \mathrm{~F}=8.184, \mathrm{p}<0.05)$, while body fat mass was excluded from the predictive model, indicating a minor effect on serum leptin levels compared with the effect of serum insulin levels.

\section{DISCUSSION}

The findings of the present study showed that 180 $\mathrm{km}$ of continuous running results in elevated serum resistin levels, reduced serum leptin levels and no significant changes in serum adiponectin and visfatin levels. Moreover, approximately 20 hours after the end of the race, serum resistin and leptin levels failed to reach pre-exercise levels, despite a clear tendency towards restoration. On the other hand, no association was recorded between the studied adipokines and cortisol levels at baseline, nor during the race and the recovery period. To the best of our knowledge, this is the first study to report on the effect of prolonged strenuous exercise and acute energy deficit on serum adipokine levels of highly trained ultra-endurance athletes.
Ultra-endurance events exert a huge impact on metabolism and energy balance, being one of the most extreme and strenuous types of exercise that an individual may undertake. Impressively, $180 \mathrm{~km}$ of distance is covered in less than $28 \mathrm{~h}$, under extreme environmental conditions (running day and night in temperatures ranging from $4^{\circ} \mathrm{C}$ at night to $30^{\circ} \mathrm{C}$ and hot sun around the finishing time).

The majority of nutrition comes from metabolism of lipid sources and exercise intensity averages $70 \%$ $\mathrm{VO}_{2}$ max or less. ${ }^{6}$ Ultra-marathon athletes have free access to fluids and food (including carbohydraterich foods and drinks), with field researches having documented an hourly caloric intake ranging from less than 100 to $430 \mathrm{kcal}$ per hour. ${ }^{6}$ The athletes should expect to finish the race with an energy intake of between $36 \%$ and $54 \%$ of energy expenditure. Since no data were obtained regarding the energy input of the athletes during the race, the energy cost of the event was assessed using reported data from similar events. Thus, the estimated energy expenditure of the ultra-endurance event examined in the present study is about $11000 \mathrm{Kcal}^{7}$ and the participants experience a mean energy deficit of at least $5000 \mathrm{Kcal}$.

\section{The effect of strenuous prolonged aerobic exercise and acute energy deficit on serum resistin levels}

The present study reports significantly elevated serum resistin levels at the end of the ultra-marathon race. Moreover, at the end of the recovery phase serum resistin levels were reduced compared to post-exercise levels, but remained significantly elevated compared to pre-race values.

The limited data available on the effect of acute intensive exercise on serum resitin levels suggest that the regulation of resistin during acute exercise could follow a duration and intensity-dependent pattern. ${ }^{8-10}$ On the other hand, controversial data exist on the effect of chronic energy restriction and eating disorders on resistin levels. ${ }^{11,12}$

Despite the significant interest generated by the discovery of resistin, very little is known about the mechanisms whereby resistin mediates its actions and metabolic effects. ${ }^{2}$ The present study reports increased serum resistin levels after strenuous prolonged aerobic exercise resulting in significant energy deficit and 
reduction in circulating insulin levels. Thus, it could be hypothesized that resistin has a regulatory role in energy homeostasis, glucose metabolism and insulin resistance. However, its role in insulin resistance has been challenged, ${ }^{13}$ while no association between serum resistin and serum insulin levels were documented in the present study.

Bearing in mind the unproven role of resistin in energy balance and glucose homeostasis, the inflammatory role of resistin could explain its regulation during acute exercise. Specifically, laboratory investigations have demonstrated that inflammatory responses, including TNF- $\alpha$ and IL-6, may stimulate resistin secretion. ${ }^{13}$ Considering that during acute exercise catecholamines stimulate the secretion of pro-inflammatory cytokines, primarily IL- 6 and TNF- $\alpha$ as well, ${ }^{1}$ an induction of resistin secretion could be expected during the strenuous prolonged exercise of the present study. Thus, further research is needed to evaluate the association of serum resistin levels with the response to exercise stress (e.g. inflammatory response, cortisol secretion).

\section{The effect of strenuous prolonged aerobic exercise and acute energy deficit on serum leptin levels}

Our results show significantly reduced serum leptin levels at the end of the ultra-marathon endurance race. At the recovery phase, serum leptin levels remained significantly lower compared to pre-race values, despite a tendency towards restoration.

The present study presents results from the most extreme, prolonged and energy-expending type of aerobic exercise and confirms the results of studies reporting on the changes in serum leptin levels during prolonged exercise..$^{14,15}$ On the other hand, several studies report no effect of exercise on serum leptin levels when the duration is too short $(\leq 60 \mathrm{~min})$ or the energy expenditure is too low $(\leq 800 \mathrm{Kcal}){ }^{4}$

Moreover, the present study reports a positive correlation between baseline leptin and insulin levels and between the changes of their levels during the race. In addition, stepwise regression analysis underscored the greater impact of insulin levels on leptin levels, in comparison with body fat mass. Interestingly, if leptin values are expressed as the ratio per kilogram $(\mathrm{kg})$ of body fat, a significant reduction is documented as well. Thus, it could be suggested that insulin mediates the regulatory role of leptin in energy homeostasis during prolonged aerobic exercise.

Our results confirm the well studied and documented association of insulin action and leptin secretion. ${ }^{16}$ Specifically, insulin stimulates both leptin biosynthesis and secretion from adipose tissue, while leptin was shown to inhibit insulin secretion in lean animals and modulates pancreatic $\beta$-cell function through direct and indirect central actions. ${ }^{16}$ In conclusion, the results of the present study confirm the role of leptin not only as an indicator of adipose stores, but as a regulator of metabolic balance and energy homeostasis.

\section{The effect of strenuous prolonged aerobic exercise and acute energy deficit on serum adiponectin levels}

The present study reports no significant changes in serum adiponectin levels at the end of the ultraendurance running race nor during the recovery phase. Thus, the combination of prolonged aerobic exercise and significant energy deficit exerts no significant effect on adiponectin levels.

To date, very few studies have provided data on the effect of short-term $(\leq 120 \mathrm{~min})$ exercise of various types (rowing, cycling and running) on serum adiponectin levels, ${ }^{4}$ producing non-conclusive results. On the other hand, serum adiponectin inversely correlates with obesity, ${ }^{2}$ while higher adiponectin levels have been documented in conditions of extreme malnourishment and severe energy deprivation. ${ }^{17,18}$

Considering the aforementioned data, the results of our study could suggest that acute physical exercise per se does not regulate serum adiponectin levels, regardless of the intensity and the duration. Furthermore, the action of adiponectin as a starvation signal and a regulator of energy homeostasis is likely exerted in conditions of chronic energy restriction rather than acutely established energy deficit.

Interestingly, 16 hours after the end of the race, serum adiponectin levels were reduced compared with the post-exercise levels. This result could be attributed to the caloric intake and the feeding pattern adopted by the athletes during the recovery 
phase. Accordingly, studies in rodents have reported that a high fat diet reduces mitochondrial function in adipocytes and down-regulates adiponectin gene expression in mesenteric adipose tissue..$^{19,20}$

\section{The effect of strenuous prolonged aerobic exercise and acute energy deficit on serum visfatin levels}

The present study reports no significant change in serum visfatin levels, neither during the running race nor during the recovery period. To date very few studies have reported on the effect of exercise on visfatin levels and the results seem rather conflicting. ${ }^{21-23}$ The results of the present study suggest that strenuous prolonged aerobic exercise and the acute energy deficit do not affect serum visfatin levels. Furthermore, Kang et $\mathrm{al}^{24}$ reported no change in circulating visfatin levels of women with hypothalamic amenorrhea due to strenuous exercise or low body weight. Thus, it could be hypothesized that visfatin is likely not involved in the metabolic and neuroendocrine abnormalities characteristic of energy regulation.

It should be noted that the aforementioned results should be evaluated also considering the diurnal variation of the examined hormones. More specifically, blood samples at baseline and at recovery were taken late in the morning ( $\sim 0800 \mathrm{hrs})$, while post-exercise blood samples were obtained early in the afternoon ( 1500hrs). However, the physiologically expected higher morning cortisol levels and higher afternoon leptin levels ${ }^{25}$ have no impact on the reported results. On the other hand, circulating adiponectin reaches peak levels late in the morning and exhibits minimal daytime variation until late in the afternoon, ${ }^{26}$ thus minimal impact on the observed results could be assumed. Finally, circulating visfatin is significantly higher in the afternoon, ${ }^{27}$ suggesting a possible downregulation by the exercise stress and/or the energy deficit, obscured by the physiologic daytime variation.

The present study has certain limitations. First of all, this is a field study carrying all the limitations of similar studies, such as the limited number of athletes enrolled, the need not to disturb already exhausted athletes with additional interventions, etc. For instance, due to such limitations, body composition parameters were not determined at the end of the recovery phase and were assessed in just 6 of the participants post-exercise. Furthermore, no data were obtained regarding the energy input of the athletes during the race and, thus, the energy cost of the event was assessed using reported data from similar events. Therefore, a replication of the study could be attempted at a future edition of the event, taking into consideration the aforementioned limitations.

On the other hand, this is the first study to report on the effect of prolonged strenuous exercise and significant acute energy deficit on serum adipokine levels of highly trained ultra-endurance athletes. In addition, the present study is unique in character, as all variables were assessed on the field of competition, while it also provides data regarding the recovery pattern of the examined adipokines.

In conclusion, strenuous ultra-endurance aerobic exercise and acute negative energy balance lead to an up-regulation of serum resistin levels, a downregulation of serum leptin levels and no change in serum adiponectin and visfatin levels. Moreover, in conditions of acute energy deficit, insulin is a major determinant of leptin variation, possibly mediating its regulatory role in energy homeostasis. Clearly, further research is required to elucidate the role of adipokines in energy regulation, metabolic balance and stress response.

\section{REFERENCES}

1. Mastorakos G, Pavlatou M, Diamanti-Kandarakis E, Chrousos GP, 2005 Exercise and the Stress System. Hormones (Athens) 4: 73-89.

2. Galic S, Oakhilla JS, Steinberg GR, 2010 Adipose tissue as an endocrine organ. Mol Cell Endocrinol 316: 129-139.

3. Sommer G, Garten A, Petzold S, et al, 2008 Visfatin/ PBEF/Nampt: structure, regulation and potential function of a novel adipokine. Clin Sci 115: 13-23.

4. Bouassida A, Chamari K, Zaouali M, Feki Y, Zbidi A, Tabka Z, 2010 Review on leptin and adiponectin responses and adaptations to acute and chronic exercise. Br J Sport Med 44: 620-630.

5. Beaumont WV, 1972 Evaluation of hemoconcentration from hematocrit measurements. J Appl Physiol 32: 712-713.

6. Getzin AR, Milner C, La Face KM, 2011 Nutrition update for the ultraendurance athlete. Curr Sports Med Rep 10: 330-339.

7. Di Prampero PE, Salvadego D, Fusi S, Grassi B, 2009 A simple method for assessing the energy cost of running during incremental tests. J Appl Physiol 107: 1068-1075. 
8. Jamurtas AZ, Theocharis V, Koukoulis G, et al, 2006 The effects of acute exercise on serum adiponectin and resistin levels and their relation to insulin sensitivity in overweight males. Eur J Appl Physiol 97: 122-126.

9. Højbjerre L, Rosenzweig M, Dela F, Bruun JM, Stallknecht B, 2007 Acute exercise increases adipose tissue interstitial adiponectin concentration in healthy overweight and lean subjects. Eur J Endocrinol 157: 613-623.

10. Varady KA, Bhutani S, Church EC, Phillips SA, 2010 Adipokine responses to acute resistance exercise in trained and untrained men. Med Sci Sports Exerc 42: 456-462.

11. Wolfe BE, Jimerson DC, Orlova C, Mantzoros CS, 2004 Effect of dieting on plasma leptin, soluble leptin receptor, adiponectin and resistin levels in healthy volunteers. Clin Endocrinol 61: 332-338.

12. Dostalova I, Kunesova M, Duskova J, Papezova H, Nedvidkova J, 2006 Adipose tissue resistin levels in patients with anorexia nervosa. Nutrition 22: 977-983.

13. Fargnoli JL, Sun Q, Olenczuk D, et al, 2010 Resistin is associated with biomarkers of inflammation while total and high-molecular weight adiponectin are associated with biomarkers of inflammation, insulin resistance, and endothelial function. Eur J Endocrinol 162: 281-288.

14. Zaccaria M, Ermolao A, Roi GS, Englaro P, Tegon G, Varnier M, 2002 Leptin reduction after endurance races differing in duration and energy expenditure. Eur J Appl Physiol 87: 108-111.

15. Leal-Cerro A, Garcia-Luna PP, Astorga R, et al, 1998 Serum leptin levels in male marathon athletes before and after the marathon run. J Clin Endocrinol Metab 83: 2376-2379.

16. Rabe K, Lehrke M, Parhofer KG, Broedl UC, 2008 Adipokines and Insulin Resistance. Mol Med 14: 741-751.

17. Modan-Moses D, Stein D, Pariente C, et al, 2007 Modulation of adiponectin and leptin during refeeding of female anorexia nervosa patients. J Clin Endocrinol Metab 92: 1843-1847.

18. Saito K, Arata S, Hosono T, et al, 2006 Adiponectin plays an important role in efficient energy usage under energy shortage. Biochim Biophys Acta 1761: 709-716.

19. Bolduc C, Yoshioka M, St-Amand J, 2010 Acute Molecular Mechanisms Responsive to Feeding and Meal Constitution in Mesenteric Adipose Tissue. Obesity (Silver Spring) 18: 410-413.

20. Garekani ET, Mohebbi H, Kraemer RR, et al, 2011 Exercise training intensity/volume affects plasma and tissue adiponectin concentrations in the male rat. Peptides 32: 1008-1012.

21. Ghanbari-Niaki A, Saghebjoo M, Soltani R, Kirwan JP, 2010 Plasma visfatin is increased after high-intensity exercise. Ann Nutr Metab 57: 3-8.

22. Frydelund-Larsen L, Akerstrom T, Nielsen S, Keller P, Keller C, Pedersen BK, 2007 Visfatin mRNA expression in human subcutaneous adipose tissue is regulated by exercise. Am J Physiol Endocrinol Metab 292: E24-E31.

23. Jürimäe J, Rämson R, Mäestu J, et al, 2009 Plasma visfatin and ghrelin response to prolonged sculling in competitive male rowers. Med Sci Sports Exerc 41: 137-143.

24. Kang ES, Magkos F, Sienkiewicz E, Mantzoros CS, 2011 Circulating vaspin and visfatin are not affected by acute or chronic energy deficiency or leptin administration in humans. Eur J Endocrinol 164: 911-917.

25. Sinha MK, Ohannesian JP, Heiman ML, et al, 1996 Nocturnal rise of leptin in lean, obese, and non-insulindependent diabetes mellitus subjects. J Clin Invest 97: 1344-1347.

26. Gavrila A, Peng CK, Chan JL, Mietus JE, Goldberger AL, Mantzoros CS, 2003 Diurnal and ultradian dynamics of serum adiponectin in healthy men: comparison with leptin, circulating soluble leptin receptor, and cortisol patterns. J Clin Endocrinol Metab 88: 2838-2843.

27. Benedict C, Shostak A, Lange T, et al, 2012 Diurnal rhythm of circulating nicotinamide phosphoribosyltransferase (Nampt/visfatin/PBEF): impact of sleep loss and relation to glucose metabolism. J Clin Endocrinol Metab 97: E218-222. 